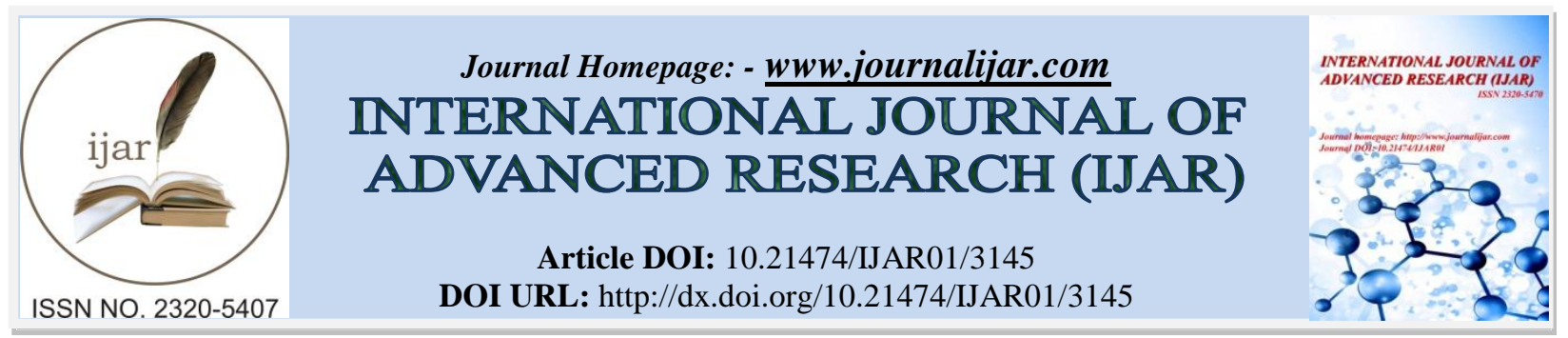

RESEARCH ARTICLE

\title{
A COMPARATIVE IN VITRO STUDY ON RETENTION AND STABILITY OF FOUR IMPLANT-SUPPORTED OVERDENTURE BASED ON IMPLANT LOCATION
}

\author{
Dr. Y. Tejaswi, Dr. A. Kaleswara rao, Dr.T. Sunil chandra, Dr. V. Ravikiran, Dr. S. Hemchand, Dr. A.
} Premalatha, Dr.T. Durgaprasad and Dr.V. Sri Harsha.

\section{Manuscript Info}

Manuscript History

Received: 18 December 2016

Final Accepted: 10 January 2017

Published: February 2017

Key words:-

Ball and socket, Dalbo, Locator,

Tensometer.

\section{Abstract}

Aim: The aim of this study is to evaluate the effect of implant location and different implant attachment systems on the retention and stability of 4 implant-supported overdentures by measuring retentive forces during vertical, oblique and horizontal types of dislodgment forces.

Materials and Methods: The retention and stability of 4 implant supported overdenture at different implant locations in the arch with different attachment forms under multidirectional dislodging forces were evaluated. For that an experiment was undertaken utilizing a model simulating a maxillary edentulous ridge with 6 dental implants in positions on the model approximating the tooth positions in the natural dentition. A cobalt-chromium cast framework with 3 loops, acrylic resin inside the housing, and chains attached to a universal testing machine was used to measure peak load $(\mathrm{N})$ required to disconnect an attachment. Kruskal wallis test followed by Mann Whitney U test were used for statistical analysis.

Results: The test results have shown significant difference $(p<0.05)$ when tested within implant location and implant attachments at different directions of pull.Kruskal wallis test was performed to find whether there is any significant difference of mean present among three attachments in each group and significant difference between three groups among each attachment system.Mann Whitney U test was done to find any significant difference present between two attachments of each group and difference between two groups among each attachment system.

Conclusion: Within the limitations of this in vitro laboratory study, the following conclusions were made. Vertical retention increased as implant location was planned at canine and premolar location. Anteroposterior stability and horizontal stability of a simulated overdenture prosthesis increased with distal implant location up to canine and premolar location. Attachment type affects retention and stability differently by location. 


\section{Introduction:-}

Edentulism is defined as loss of all permanent teeth. ${ }^{1}$ Tooth loss is a multifactorial process. ${ }^{2}$ while the rate of edentulism has been decreasing throughout the past three decades, the subsequent increase in the world population has resulted in an increasing growth of total edentulous persons. ${ }^{3}$ Residual ridge resorption continues to be primary complication of edentulism. To minimize loss of residual ridge, exemplary complete denture therapy along with routine recall systems, should be goal of the treatment. ${ }^{2}$ The traditional treatment modality of edentulism has been the fabrication of removable, tissue-supported complete dentures. ${ }^{3}$ Three main factors are involved in optimal denture treatment: retention, support and stability. ${ }^{4}$ It is critical to evaluate and properly estimate the importance of each of these three factors involved in the treatment. In choosing between treatment approaches, patients often choose the treatment that increases stability even when cost is a major factor. ${ }^{5}$ One of the greatest challenges facing the clinician is to provide a removable prosthesis with adequate retention and stability. Amount of residual ridge is correlated with the retention and stability of the denture. ${ }^{6-10}$

Overdentures have been advocated as a means to preserve the structures associated with denture support which may augment retention and stability. ${ }^{5}$ In contrast to the mandible, implant placement within maxilla may be limited by the anatomy, available bone and its relationship to the sinus architecture. Less favourable mid-term and long-term survival and success rates were originally reported for maxillary implants supporting overdentures. Compared with the mandible, the maxilla contains softer bone and a different distribution of occlusal forces. Therefore, a greater number of implants are required in the maxilla compared with the mandible. However, there are no specific guidelines for the number of implants necessary to support a maxillary overdenture. A minimum of four well-spaced implants is often recommended for an implant-supported and -retained overdenture. Many studies have demonstrated that the use of only four maxillary implants can successfully restore oral function for completely

maxillary edentulous patients. Evidence has supported the treatment philosophy of focusing on providing proper standard of care within the realms of simplicity versus overly complicated. The use of stud-style attachments is considered a simplified and cost-effective treatment as compared to bar and clip type implant overdentures. While many of these studies focus their attention on the retention, release, and stability between types and forms of attachments, few have investigated overdenture properties as a function of attachment location, distribution, and number of implants. The impact of distribution and number of implants and attachment systems upon retention and stability of overdentures has been alluded to in several studies. In consideration of the currently available studies, limited information exists regarding implant position, distribution, and number and the effect upon the retention and stability of maxillary implant overdentures. The purpose of this investigation was to provide an in vitro evaluation of retention and stability of four implant supported overdentures based on implant location.

\section{Materials and Method:-}

An experiment was undertaken utilizing a model simulating a maxillary edentulous ridge with 6 dental implants in positions on the model approximating the tooth positions in the natural dentition. A cobalt-chromium cast framework with 3 loops, acrylic resin inside the housing, and chains attached to a universal testing machine was used to measure peak load $(\mathrm{N})$ required to disconnect an attachment. Three different types of attachments were used in 3 positions on the model in sequence of 4 implants at a time.

Auto polymerizing polymethyl methacrylate (PMMA) acrylic resin was incorporated in the intaglio and facial/lingual surfaces of the framework to allow for attachment of the matrix portions. The metal framework remained constant throughout testing. Three commercially available attachment designs were evaluated: Ball and socket, Locator, Dalbo attachments. The occlusal plane of the test model was set even with the horizontal plane of a metal plate and 3 bolts were placed to affix the model to the metal plate. The incorporation of the plate allows precise reproduction of the position of the model clamping to the testing apparatus for the different attachment systems. A universal testing machine (Tensometer) was applied to test forces required to dislodge the prosthesis in various directions as described previously. Three $6.2 \mathrm{~cm}$ metal chains were attached to a washer with three eye bolts in a triangular orientation with machine screw nuts. The washer was attached in the center with bolt and nut to a ball/socket pivoting joint assembly incorporated into the universal testing machine. The use of the eye bolts and pivoting joint allowed for precise adjustment of the chains and to ensure that all chains were pulling evenly throughout the experiment. The testing machine instrumentation was calibrated and balanced using the testing machine's computer algorithm to account for the weight of the simulated prosthesis and chains. Three chains were attached to the prosthesis and a 3-point vertical pull was used to determine retention against a vertically directed 
dislodging force parallel to the path of insertion. A 2-point oblique/posterior pull was used to determine stability to determine resistance against Para-axial, oblique dislodging forces. Two chains were attached: one in incisor region and alternating chains either on the right or left side molar region. To test posterior dislodging forces, the incisor chain was removed and the remaining two chains were attached in the molar regions. The chains were adjusted to reduce slack and force was applied until separation of the prosthesis occurred. The dislodging force applied resulted in a peak load measurement (Newton's, N). For each group, 9 measurements were made of peak dislodging forces. Means were calculated and differences among the systems, directions, and groups were identified. Four patrix portions of the attachment system were placed into areas designed as group numbers that approximate natural tooth positions: Group A (\#11,13,21,23), Group B (\#13,14,23,24), Group C (\#11,14,21,24). Matrix housing portions of the attachment system were attached to the prosthesis following manufacturer guidelines with a bis-acryl material.

\section{Results:-}

The present study was done to evaluate and compare the retention and stability of four implant supported overdentures based on implant location. In this study retention and stability of an implant overdenture was tested between different implant locations as group A $(\# 11,13,21,23)$, group B $(\# 13,14,23,24)$, group C (\#11,14,21,24) and different attachment systems using tensometer. The following results of statistical analysis have been obtained in this study using SPSS software package. Descriptive statistics for the three groups and different attachments were tabulated in table $1,2,3,4,5$ and 6 respectively.The test results have shown significant difference $(p<0.05)$ when tested within implant location and implant attachments at different directions of pull.

Kruskal wallis test was performed to find whether there is any significant difference of mean present among three attachments in each group and significant difference between three groups among each attachment system. Mann Whitney $\mathbf{U}$ test was done to find any significant difference present between two attachments of each group and difference between two groups among each attachment system. Table1 and graph1- Shows the mean and standard deviation of different attachments among group A. The maximum mean value is of dalbo attachment (43.5N) at vertical pull. The minimum mean value is of locator attachment $(14.4 \mathrm{~N})$ at oblique pull. Ball and locator attachments showed significant difference only at vertical and oblique pull $(\mathrm{p}<0.05)$. The results were not significant between ball and locator attachments at posterior pull ( $\mathrm{p}>0.05)$. Table2 and graph2- Shows the mean and standard deviation of different attachments among group B.The maximum mean value is of dalbo attachment (59.8N) at posterior pull. The minimum mean value is of locator attachment $(13.8 \mathrm{~N})$ at oblique pull. Ball and locator attachments showed significant difference only at vertical and posterior pull $(\mathrm{p}<0.05)$. The results were not significant between ball and locator attachments at oblique pull ( $p>0.05)$. Table3 and graph3- Shows the mean and standard deviation of different attachments among group $\mathrm{C}$. The maximum mean value is of dalbo attachment $(42.5 \mathrm{~N})$ at vertical pull. The minimum mean value is of locator attachment $(15.5 \mathrm{~N})$ at oblique pull.. Ball and locator attachments showed significant difference only at vertical and oblique pull $(\mathrm{p}<0.05)$. The results were not significant between ball and locator attachments at posterior pull $(\mathrm{p}>0.05)$. Table4 and graph4- Shows the mean and standard deviation of Ball and socket attachment among different groups'. The maximum mean value is at group B (34.94N) at vertical pull. The minimum mean value is at group B (19.64N) at oblique pull. Table5 and graph5- Shows the mean and standard deviation of Dalbo attachment among different groups. The maximum mean value is at group B $(59.78 \mathrm{~N})$ at posterior pull. The minimum mean value is at group C $(35.60 \mathrm{~N})$ at oblique pull. Kruskal Wallis test was performed to find whether there was significant difference between three groups among dalbo attachment, results showed there is statistically significant $(\mathrm{p}<0.05)$ difference among group A, B and C at posterior direction of pull. Table6 and graph6- Shows the mean and standard deviation of Locator attachment among different groups .The maximum mean value is at group $\mathrm{C}(19.08 \mathrm{~N})$ at posterior pull. The minimum mean value is at group B (13.84N) at oblique pull.

\section{Discussion:-}

It is evident that many treatment concepts involving overdentures are based on empirical experiences of individuals. Clinicians often base their selection of implant location and attachment system empirically on expected retentive qualities. Evaluating these factors the results allow the clinician to formulate a comparison of implant location to retention and stability of implant-retained overdenture prosthesis.

While retention and its effect upon overdenture prosthetic factors are related, few studies have established a consensus regarding what is considered sufficient retention. Zou et al The results of prospective study clearly indicate favorable outcomes for four interconnected implants supporting a maxillary overdenture with three different 
abutments. This study was an effective measure of clinical factors related to prosthetic success and acceptance by the patients at several time points throughout treatment and patients preferred the attachment that provided greater retention. Based upon these studies, it can be established that an effective retentive force may be between 8 to $10 \mathrm{~N}$. Overdentures, when in place in the oral environment, move in complex ways. Movement of overdentures typically occurs in six directions: occlusal, gingival, mesial, distal, facial, and lingual. While true uni-directional dislodging forces rarely occur in clinical scenarios, directional pull-testing is an effective way of measuring retention and stability of a prosthesis during in vitro laboratory evaluation. The present in vitro study investigated the effect of implant position on the retention and stability of a simulated prosthesis. To the knowledge, no studies have evaluated the effect of varying in vitro implant location upon dislodging forces of simulated 4-implant Maxillary overdenture prosthesis. The results of this study indicate that implant location affects in vitro retention and stability of an implant overdenture. The current in vitro study reveals that vertical retention increases with implant location up to premolar. Regarding vertically directed forces, one would believe that retentive values would not change when implant location was modified. In the testing procedures, it was noted that during 3-point chain pull tests, some antero-posterior movement occurred. While this may have affected the reported force values, the method employed better simulates the movement of overdentures in clinical situations rather than utilizing a rigid design. The type of attachment affects the effect of vertically applied forces. Horizontal stability of Dalbo attachment was affected by implant positioning, incisor and canine sites had the highest values. In the present study, antero-posterior chain pulls were evaluated as an indirect method of determining the effect of implant location upon posterior dislodging forces. In all attachments systems tested, a general trend was determined that an increased resistance to dislodgment occurred as implant location was moved distally. Interestingly, all systems except the Locator group showed significant increase in resistance in moving implant location. The variation between attachment systems is of great interest when formulating conclusions regarding the effect of implant position upon retention and stability. The present study shows that attachment type affects retention and stability different by location. The results of this study illustrate that attachment systems respond in different ways depending on their location in the edentulous arch.

The results of this study indicate that 4-implants may produce effective in vitro retention and stability of overdenture prosthesis. The testing performed is limited with specific conditions and methods and does not completely replicate clinical situations as the implant overdenture clinical reality is much more complex than a laboratory setting can replicate. Furthermore, the findings of this study also do not account for attachment wear, resiliency, and tissue effects. While this in vitro based analysis shows a statistical difference between groups, long-term comparative prospective controlled studies are needed to reach agreement on an accepted treatment concept. Factors such as the type and location of implants placed, quality and quantity of bone, and type of superstructure should be part of these studies. In evaluating implant location for implant-retained overdentures, it is important to consider the biomechanics of how the prosthesis functions. Previously considered in relation to removable partial denture design, Avant described the effect of indirect retainers upon the mechanical advantage of a distal extension base. The indirect retainer acts as the fulcrum, the direct retainer assembly acts as the resistance, and the power is the force that lifts the denture base away from the ridge. Avant described methods of lowering the mechanical advantage of the lever in order to keep the denture base from lifting away from the ridge. Determination of mechanical advantage is performed by measuring the ratio of the power arm to the resistance arm. When considering antero-posterior movement such as a dislodging force would provide, an implant overdenture may function as a class I, II or III lever. Fig. Components of a Class I, II and III lever system with the implant and attachment serving as the resistance / fulcrum point. Assuming the example of an implant-retained overdenture prosthesis that is intimately fitting the soft tissue support, the fulcrum is the anterior alveolar ridge, the resistance is the attachment system, and the power is the posterior dislodging force lifting the denture base away from the ridge. Analyzing an example where implant location is anterior, such as in the incisor region, figure (A) illustrates a class I lever system. The fulcrum and resistance point would be coincident, thus making for a short resistance arm. Moving implant location distally, such as in the 1st premolar location, as shown in figure (B), the resistance arm is substantially lengthened compared to that shown in figure 16 (A). This change would also modify the lever system to a class II. Fig. Components of a Class I, II lever system with the anterior residual alveolar ridge as the fulcrum point, implant and attachment serving as the resistance and power is the posterior dislodging force.The power required to exact a similar dislodging force would be much higher in example (B) due to the reduction in mechanical advantage by lengthening the resistance arm. This mechanical illustration may help explain the results of this study. As seen in the analysis of the anteroposterior dislodging force test, significantly higher forces were required to dislodge the prosthesis when implants were located distally on the test model. 


\section{Summary and Conclusion:-}

The aim of this study was to evaluate the effect of implant location and different implant attachments on the retention and stability of implant-supported overdentures by measuring retentive forces during vertical, oblique and horizontal types of dislodgment forces.

The results of statistical analysis obtained in this study using SPSS software. Within the limitations of this in vitro laboratory study, the following conclusions were made.

The interactions between attachment system, direction of force, and implant location were statistically significant ( $p$ $<0.005)$.

1. Vertical retention increased as implant location was placed at canine and premolar location.

2. Antero-posterior stability and horizontal stability of a simulated overdenture prosthesis increased with distal implant location up to canine and premolar location.

3. Attachment type affects retention and stability differently by location

Table 1:- Comparison of Mean values and standard deviation of 3attachment systems in vertical, oblique and anteroposterior direction among Group A in Newtons

\begin{tabular}{|c|c|c|c|c|c|c|c|c|c|c|c|c|}
\hline \multirow[t]{2}{*}{ Group-A } & \multicolumn{4}{|c|}{ Vertical } & \multicolumn{4}{|c|}{ Oblique } & \multicolumn{4}{|c|}{ Posterior } \\
\hline & Min & Max & Mean & SD & Min & Max & Mean & SD & Min & Max & Mean & SD \\
\hline Ball & 24.3 & 27.3 & 25.9 & 1.5 & 18.7 & 21.7 & 19.9 & 1.6 & 18.5 & 20.8 & 19.8 & 1.2 \\
\hline Dalbo & 42.5 & 44.6 & 43.5 & 1.1 & 36.9 & 38.3 & 37.8 & 0.8 & 38.8 & 42.3 & 40.4 & 1.8 \\
\hline Locator & 15.2 & 21.3 & 18.3 & 3.1 & 12.2 & 17.4 & 14.4 & 2.7 & 13.5 & 19.3 & 16.0 & 3.0 \\
\hline B vs D vs L & \multicolumn{4}{|c|}{0.03} & \multicolumn{4}{|c|}{0.03} & \multicolumn{4}{|c|}{0.04} \\
\hline B vs D & \multicolumn{4}{|c|}{0.05} & \multicolumn{4}{|c|}{0.05} & \multicolumn{4}{|c|}{0.05} \\
\hline B vs L & \multicolumn{4}{|c|}{0.05} & \multirow{2}{*}{\multicolumn{4}{|c|}{0.05}} & \multicolumn{4}{|c|}{0.13} \\
\hline D vs L & \multicolumn{4}{|c|}{005} & & & & & \multicolumn{4}{|c|}{005} \\
\hline
\end{tabular}

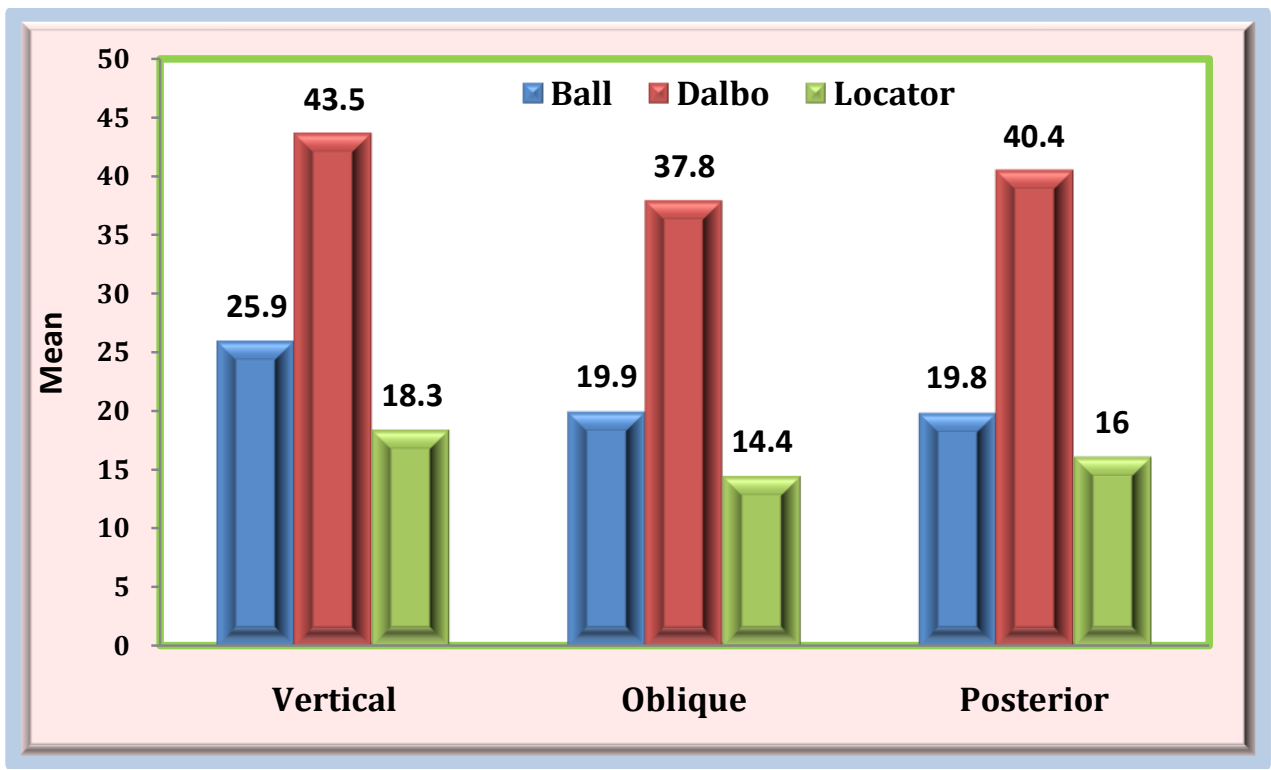

Diagram 1:- Mean Comparison of 3attachment systems in vertical, oblique and anteroposterior direction among Group A. 
Table 2:- Comparison of Mean values and standard deviation of 3attachment systems in vertical, oblique and anteroposterior direction among Group B in Newtons

\begin{tabular}{|c|c|c|c|c|c|c|c|c|c|c|c|c|}
\hline \multirow[t]{2}{*}{ Group-B } & \multicolumn{4}{|c|}{ Vertical } & \multicolumn{4}{|c|}{ Oblique } & \multicolumn{4}{|c|}{ Posterior } \\
\hline & Min & Max & Mean & SD & Min & Max & Mean & SD & Min & Max & Mean & SD \\
\hline Ball & 33.1 & 37.3 & 34.9 & 2.2 & 16.8 & 22.3 & 19.6 & 2.7 & 27.6 & 34.2 & 30.5 & 3.4 \\
\hline Dalbo & 40.2 & 44.3 & 42.0 & 2.0 & 53.3 & 59.7 & 56.3 & 3.2 & 57.4 & 62.2 & 59.8 & 2.4 \\
\hline Locator & 14.4 & 22.5 & 18.0 & 4.2 & 11.8 & 17.7 & 13.8 & 3.3 & 14.0 & 20.7 & 16.5 & 3.7 \\
\hline B vs D vs L & \multicolumn{4}{|c|}{0.03} & \multicolumn{4}{|c|}{0.04} & \multicolumn{4}{|c|}{0.03} \\
\hline B vs D & \multicolumn{4}{|c|}{0.05} & \multicolumn{4}{|c|}{0.05} & \multicolumn{4}{|c|}{0.05} \\
\hline B vs L & \multirow{2}{*}{\multicolumn{4}{|c|}{0.05}} & \multicolumn{4}{|c|}{0.13} & \multicolumn{4}{|c|}{0.05} \\
\hline D vs L & & & \multicolumn{2}{|c|}{0.05} & \multicolumn{4}{|c|}{0.05} & \multicolumn{4}{|c|}{0.05} \\
\hline
\end{tabular}

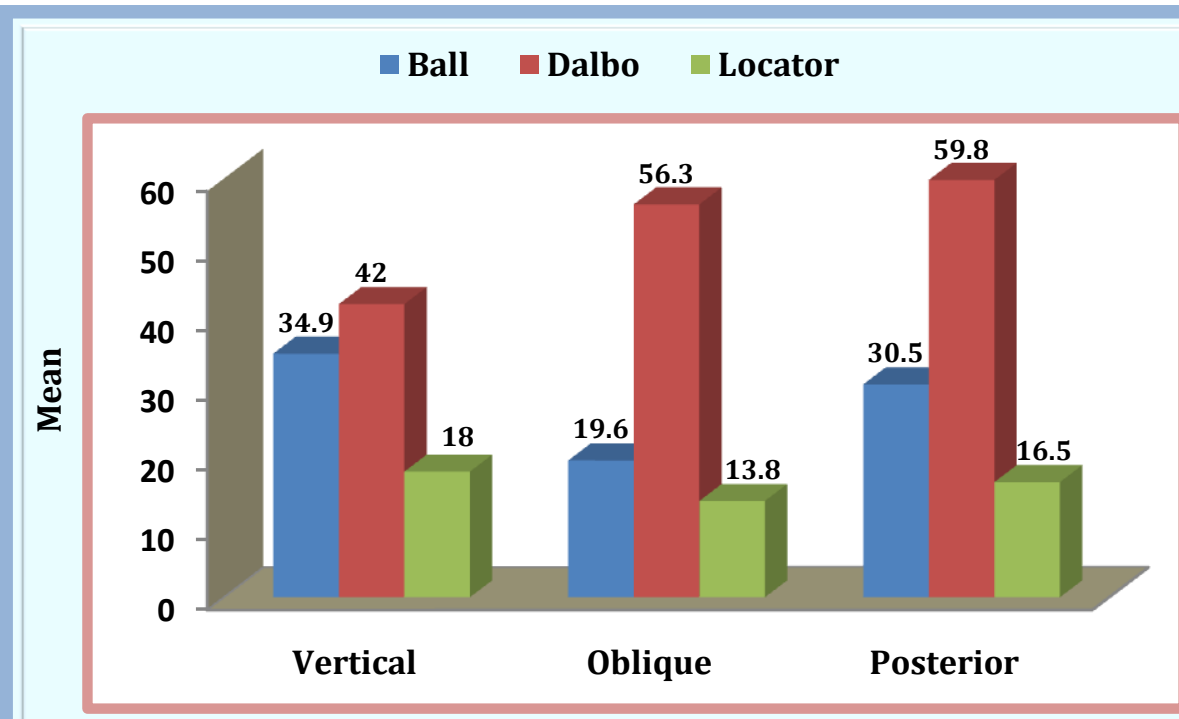

Diagram 2:- Mean Comparison of 3attachment systems in vertical, oblique and anteroposterior direction among Group B.

Table 3:- Comparison of Mean values and standard deviation of 3attachment systems in vertical, oblique and anteroposterior direction among Group C in Newtons

\begin{tabular}{|c|c|c|c|c|c|c|c|c|c|c|c|c|}
\hline \multirow[t]{2}{*}{ Group-c } & \multicolumn{4}{|c|}{ Vertical } & \multicolumn{4}{|c|}{ Oblique } & \multicolumn{4}{|c|}{ Posterior } \\
\hline & Min & Max & Mean & SD & Min & Max & Mean & SD & Min & Max & Mean & SD \\
\hline Ball & 30.2 & 35.7 & 32.7 & 2.8 & 19.7 & 22.3 & 21.0 & 1.3 & 20.3 & 25.4 & 23.4 & 2.7 \\
\hline Dalbo & 40.6 & 43.8 & 42.5 & 1.7 & 32.6 & 39.4 & 35.6 & 3.5 & 35.5 & 37.9 & 36.9 & 1.3 \\
\hline Locator & 15.5 & 20.9 & 18.1 & 2.7 & 12.5 & 18.4 & 15.5 & 3.0 & 14.4 & 22.2 & 19.1 & 4.1 \\
\hline B vs D vs L & \multicolumn{4}{|c|}{0.03} & \multicolumn{4}{|c|}{0.03} & \multicolumn{4}{|c|}{0.05} \\
\hline B vs D & \multicolumn{4}{|c|}{0.05} & \multicolumn{4}{|c|}{0.05} & \multicolumn{4}{|c|}{0.05} \\
\hline B vs L & \multicolumn{4}{|c|}{0.05} & \multicolumn{4}{|c|}{0.05} & \multicolumn{4}{|c|}{0.28} \\
\hline D vs L & \multicolumn{4}{|c|}{0.05} & \multicolumn{4}{|c|}{0.05} & \multicolumn{4}{|c|}{0.05} \\
\hline
\end{tabular}




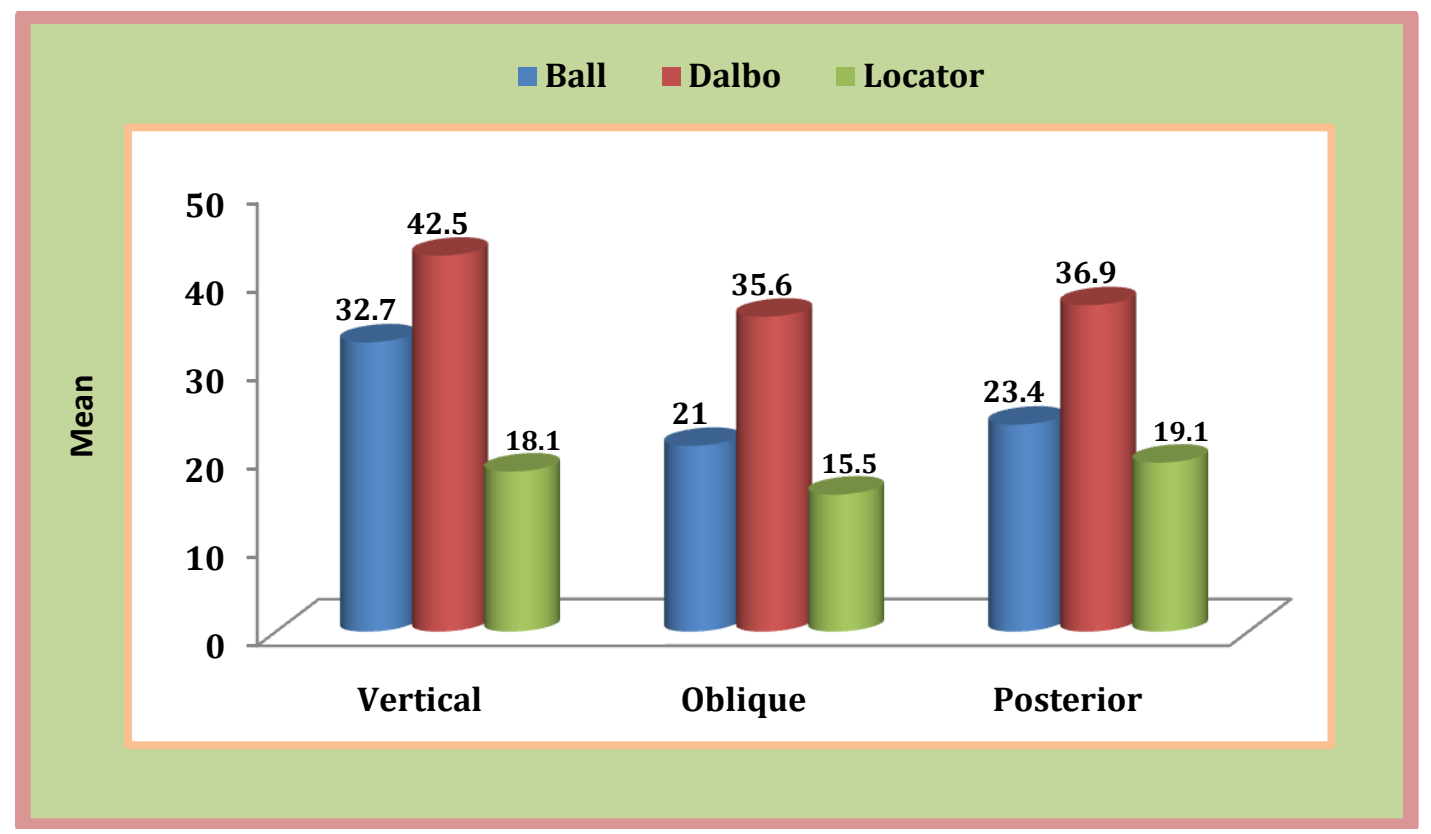

Diagram 3:- Mean Comparison of 3attachment systems in vertical, oblique and anteroposterior direction among Group C.

Table 4:- Comparison of Mean values and standard deviation of Ball attachment in vertical, oblique and anteroposterior direction among different groups in Newtons

\begin{tabular}{|c|c|c|c|c|c|c|}
\hline \multirow{2}{*}{ Ball } & \multicolumn{2}{|c|}{ Vertical } & \multicolumn{2}{c|}{ Oblique } & \multicolumn{2}{c|}{ Posterior } \\
\cline { 2 - 7 } & Mean & SD & Mean & SD & Mean & SD \\
\hline Group A & 25.92 & 1.55 & 19.94 & 1.56 & 19.85 & 1.16 \\
\hline Group B & 34.94 & 2.16 & 19.64 & 2.74 & 30.54 & 3.37 \\
\hline Group C & 32.66 & 2.75 & 20.99 & 1.34 & 23.36 & 2.69 \\
\hline A vs B vs C & \multicolumn{2}{|c|}{0.05} & \multicolumn{2}{|c|}{0.56} & \multicolumn{2}{c|}{0.04} \\
\hline A vs B & \multicolumn{2}{|c|}{0.05} & - & \multicolumn{2}{c|}{0.05} \\
\hline A vs C & \multicolumn{2}{|c|}{0.05} & - & \multicolumn{2}{c|}{0.05} \\
\hline B vs C & \multicolumn{2}{|c|}{0.23} & \multicolumn{2}{c|}{} \\
\hline
\end{tabular}

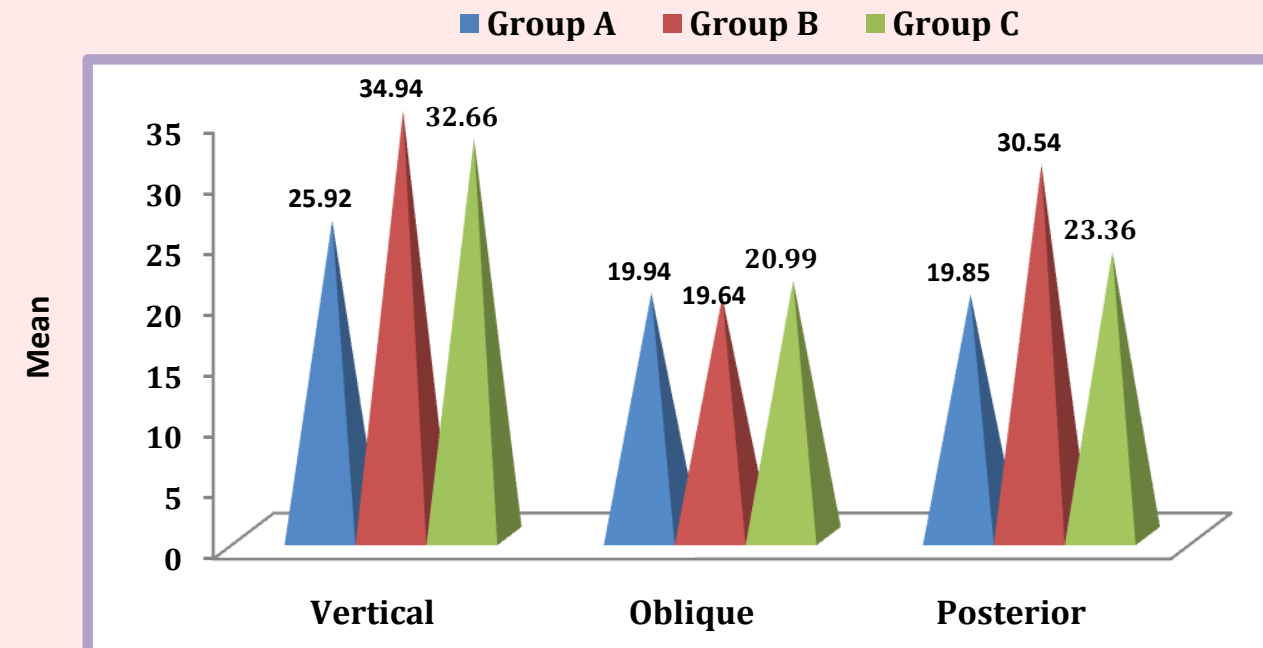

Diagram 4:- Mean Comparison of Ball attachment in vertical, oblique and anteroposterior direction among different groups. 
Table 5:- Comparison of Mean values and standard deviation of Dalbo attachment in vertical, oblique and anteroposterior direction among different groups in Newtons

\begin{tabular}{|c|c|c|c|c|c|c|}
\hline \multirow[t]{2}{*}{ Dalbo } & \multicolumn{2}{|c|}{ Vertical } & \multicolumn{2}{|c|}{ Oblique } & \multicolumn{2}{|c|}{ Posterior } \\
\hline & Mean & SD & Mean & SD & Mean & SD \\
\hline Group A & 43.49 & 1.08 & 37.81 & 0.82 & 40.41 & 1.75 \\
\hline Group B & 42.05 & 2.05 & 56.27 & 3.23 & 59.78 & 2.36 \\
\hline Group C & 42.53 & 1.73 & 35.60 & 3.48 & 36.93 & 1.29 \\
\hline $\mathrm{A}$ vs $\mathrm{B}$ vs $\mathrm{C}$ & \multicolumn{2}{|c|}{0.56} & \multicolumn{2}{|c|}{0.06} & \multicolumn{2}{|c|}{0.03} \\
\hline $\mathrm{A}$ vs B & \multicolumn{2}{|c|}{-} & \multicolumn{2}{|c|}{-} & \multicolumn{2}{|c|}{0.05} \\
\hline A vs $\mathrm{C}$ & \multicolumn{2}{|c|}{-} & \multicolumn{2}{|c|}{-} & \multicolumn{2}{|c|}{0.05} \\
\hline $\mathrm{B}$ vs $\mathrm{C}$ & \multicolumn{2}{|c|}{-} & \multicolumn{2}{|c|}{-} & \multicolumn{2}{|c|}{0.05} \\
\hline
\end{tabular}

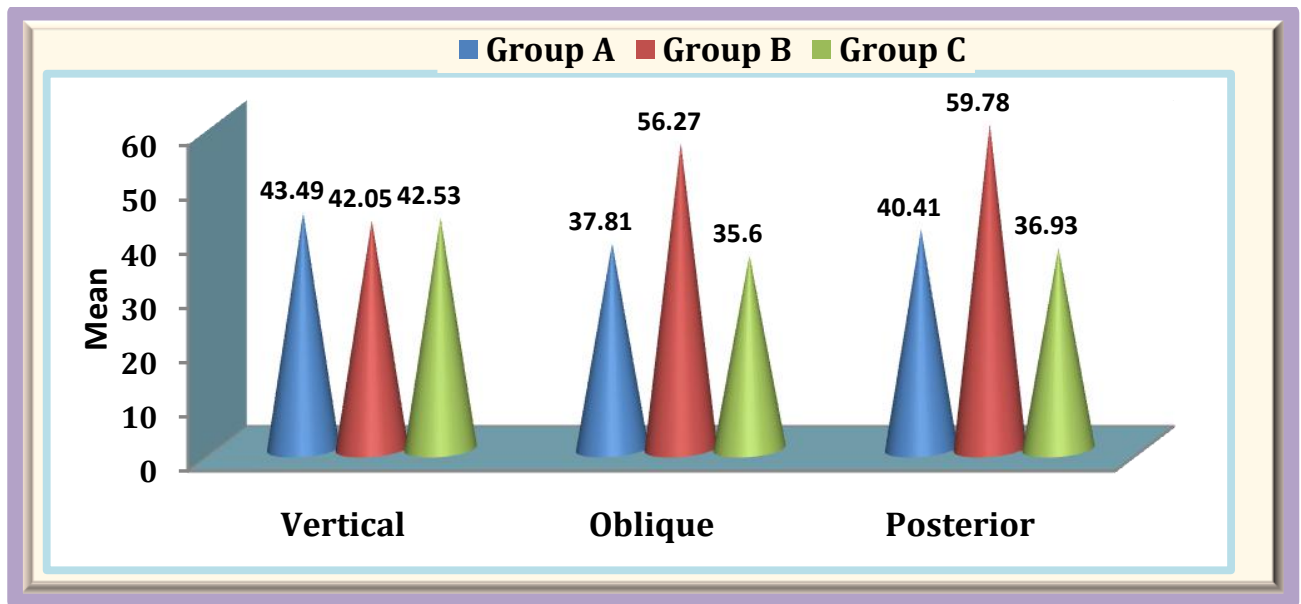

Diagram 5:- Mean Comparison of Dalbo attachment in vertical, oblique and anteroposterior direction among different groups.

Table 6:- Comparison of Mean values and standard deviation of Locator attachment in vertical, oblique and anteroposterior direction among different groups in Newtons

\begin{tabular}{|c|c|c|c|c|c|c|}
\hline \multirow{2}{*}{ Locator } & \multicolumn{2}{|c|}{ Vertical } & \multicolumn{2}{c|}{ Oblique } & \multicolumn{2}{c|}{ Posterior } \\
\cline { 2 - 7 } & Mean & SD & Mean & SD & Mean & 2.98 \\
\hline Group A & 18.27 & 3.07 & 14.36 & 2.72 & 16.01 & 3.67 \\
\hline Group B & 17.95 & 4.18 & 13.84 & 3.30 & 16.45 & 4.11 \\
\hline Group C & 18.07 & 2.74 & 15.46 & 2.99 & 19.08 & \multicolumn{2}{|c|}{0.56} \\
\hline A vs B vs C & \multicolumn{3}{|c|}{0.96} & \multicolumn{2}{|c|}{0.49} & \multicolumn{2}{|c|}{} \\
\hline
\end{tabular}

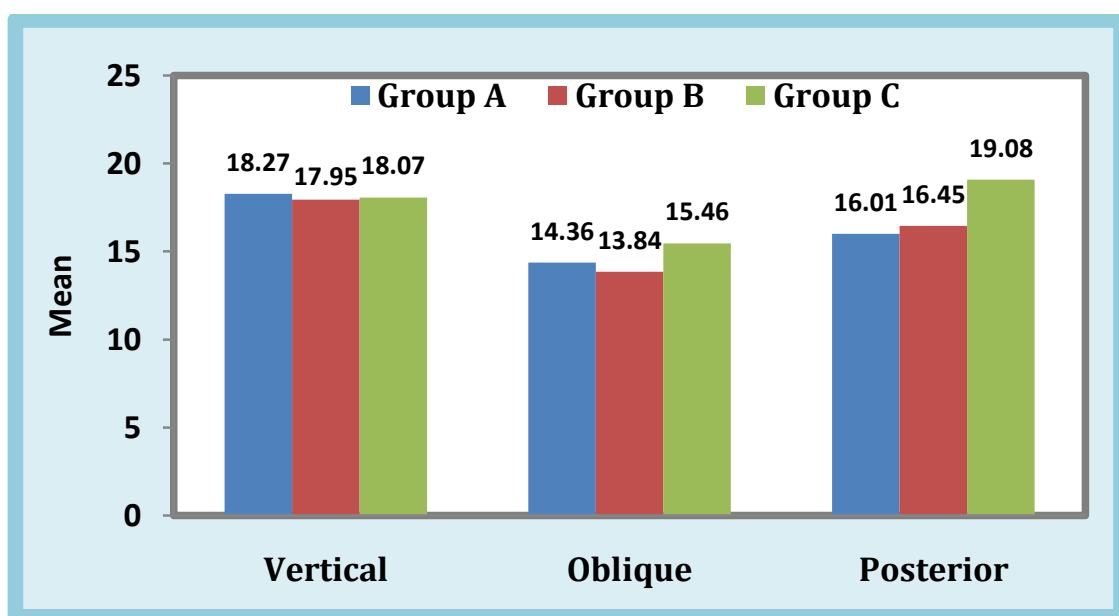

Diagram 6:- Mean Comparison of Locator attachment in vertical, oblique and anteroposterior direction among different groups. 


\section{References:-}

1. Academy of prosthodontics: glossary of prosthodontic terms. J Prosthet Dent 2005;94:10-92 .

2. Felton DA. Edentulism and comorbid factors.J Prosthodont. 2009; 18:88-96.

3. Carlsson GE, Omar R. The future of complete dentures in oral rehabilitation. A critical review. J Oral Rehabil. 2010; 37:143-56.

4. Lang BR. A review of traditional therapies in complete dentures. J Prosthet Dent.1994; 72:538-42.

5. Jacobson TE, krol AJ. A contemporary review of the factors involved in complete denture retention, stability, and support. Part i: retention. J Prosthet Dent.1983; 49:5-15.

6. Mericske-stern R, Probst D, Fahrländer F, Schellenberg M. Within-subject comparison of two rigid bar designs connecting two interforaminal implants: patients' satisfaction and prosthetic results. Clin implant dent relat res. 2009; 11:228-37.

7. Carlsson GE, Otterland A, Wennstrom A. Patient factors in appreciation of complete dentures. J Prosthet Dent. 1967;17:322- 328 .

8. Gjengedal H, Berg E, Boe OE, Trovik TA. Self-reported oral health and denture satisfaction in partially and completely edentulous patients. Int J Prosthodont. 2011; 24:9-15.

9. Assunçao WG, Barao VA, Delben JA, Gomes EA, Tabata LF. A comparison of patient satisfaction between treatment with conventional complete dentures and overdentures in the elderly: a literature review. Gerodontology. 2010; 27:154-62.

10. Critchlow SB, Ellis JS. Prognostic indicators for conventional complete denture therapy: a review of the literature. J Dent. 2010; 38:2-9.

11. Zou et al. A 3-year prospective clinical study of telescopic crown, bar, and locator attachments for removable four implant-supported maxillary overdentures. Int J Prosthodont 2013; 26:566-573.

12. Sadowsky SJ. Mandibular implant-retained overdentures: a literature review. J Prosthet ent. 2001; 86:468-73.

13. Trakas T, Michalakis K, Kang K, Hirayama H. Attachment systems for implant retained overdentures: a literature review. Implant Dent. 2006;15:24-34

14. Alsabeeha NH, Payne AG, Swain MV. Attachment systems for mandibular two-implant overdentures: a review of in vitro investigations on retention and wear features. Int J Prosthodont. 2009; 22:429-40.

15. Brewer AA, Morrow RM. Examination, diagnosis, treatment planning, and prognosis. In overdentures (pp. 3236) 1975. St. Louis: C.V. mosby co.

16. Langer Y, Langer A. Root-retained overdentures: part i--biomechanical and clinical aspects. J prosthet dent. 1991; 66:784-9.

17. Kaufmann R, Friedli M, Hug S, Mericske-stern R. Removable dentures with implant support in strategic positions followed for up to 8 years. Int J Prosthodont. 2009; 22:233-41.

18. Zitzmann NU, Rohner U, Weiger R, Krastl G. When to choose which retention element to use for removable dental prostheses. Int J Prosthodont. 2009; 22:161-7.

19. Mumcu E, Bilhan H, Geckili O. The effect of attachment type and implant number on satisfaction and quality of life of mandibular implant-retained overdenture wearers. Gerodontology. 2011;jul:4:1-6 